\title{
Universal Bounds on Fluctuations in Continuous Thermal Machines
}

\author{
Sushant Saryal, ${ }^{1}$ Matthew Gerry, ${ }^{2}$ Ilia Khait, ${ }^{2}$ Dvira Segal, ${ }^{3,2}$ and Bijay Kumar Agarwalla ${ }^{1}$ \\ ${ }^{1}$ Department of Physics, Indian Institute of Science Education and Research, Pune 411008, India \\ ${ }^{2}$ Department of Physics, University of Toronto, Toronto, Ontario, Canada M5S $1 A 7$ \\ ${ }^{3}$ Department of Chemistry and Centre for Quantum Information and Quantum Control, \\ University of Toronto, 80 Saint George St., Toronto, Ontario, M5S 3H6, Canada
}

(Dated: March 26, 2021)

\begin{abstract}
We study bounds on ratios of fluctuations in steady-state time-reversal heat engines controlled by multi affinities. In the linear response regime, we prove that the relative fluctuations (precision) of the output current (power) is always lower-bounded by the relative fluctuations of the input current (heat current absorbed from the hot bath). As a consequence, the ratio between the fluctuations of the output and input currents are bounded both from above and below, where the lower (upper) bound is determined by the square of the averaged efficiency (square of the Carnot efficiency) of the engine. The saturation of the lower bound is achieved in the tight-coupling limit when the determinant of the Onsager response matrix vanishes. Our analysis can be applied to different operational regimes, including engines, refrigerators, and heat pumps. We illustrate our findings in two types of continuous engines: two-terminal coherent thermoelectric junctions and three-terminal quantum absorption refrigerators. Numerical simulations in the far-from-equilibrium regime suggest that these bounds apply more broadly, beyond linear response.
\end{abstract}

Introduction.- The second law of thermodynamics plays a foundational role in many branches of science $[1,2]$. It provides an upper bound on the performance of engines and prohibits the possibility of designing an ideal engine with $100 \%$ conversion efficiency, from wasted heat to useful work [1]. Significant interest is currently devoted to realizing efficient, small-scale engines with classical or quantum working fluids, made from e.g. colloidal particles, few level discrete-energy systems realized in collections of spins or quantum dots, atoms or ions [3-9]. Notably, small-scale engines may suffer from significant fluctuations (thermal and possibly quantum), which cannot be ignored when analyzing their performance. Unlike equilibrium conditions, fluctuations in nonequilibrium systems are more difficult to quantify due to the failure of the fluctuation-dissipation relation [1012]. The rapidly-growing fields of stochastic and quantum thermodynamics [13-18] have significantly enhanced our fundamental understanding of nonequilibrium fluctuations $[19,20]$ with practical ramifications such as in the application of electronic noise to measure molecular properties $[21,22]$. The discovery of fluctuation relations $[14,15,17,23-25]$ provided the underlying connection between macroscopic thermodynamics and the thermodynamics of small systems.

Recent progress in stochastic thermodynamics concerned the derivation of bounds on relative fluctuations for observables beyond equilibrium, expressed as tradeoff relations between precision (relative fluctuation) and cost (entropy production), now referred to as "Thermodynamic Uncertainty relations (TUR)" [26-34]. The TUR further constrains the performance of a thermal engine, providing a tradeoff between output power, power fluctuations and the engine's efficiency [27]. It was recently shown in Ref. [35] that for finite-time four-stroke heat en- (a)

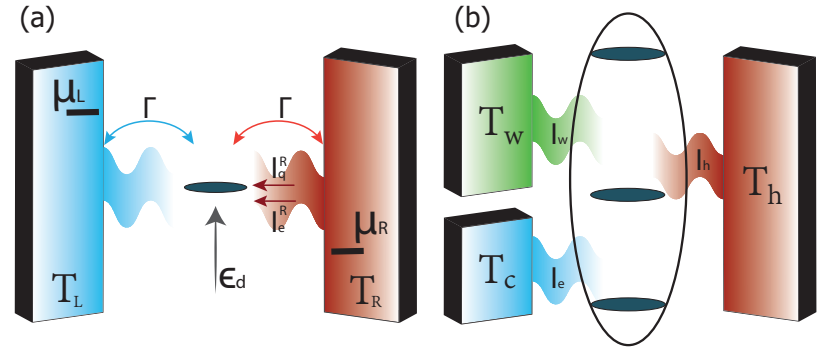

FIG. 1. (a) Illustration of a single-dot (energy $\epsilon_{d}$ ) thermoelectric engine with chemical potentials $\mu_{L, R}$ and temperature $T_{L, R}$. Horizontal arrows indicate the positive direction of currents in the engine, $\Gamma$ is the system-bath coupling strength. (b) Illustration of a three-level quantum absorption refrigerator; $I_{w, c, h}$ are the heat currents flowing from the baths $(\mathrm{R}, \mathrm{L})$ to the system. Other parameters are defined and visualized in Fig. 4.

gines, the ratio between fluctuations of output work $(W)$ and input heat $(Q)$ is upper bounded, and the bound solely depends on the temperatures of the hot $\left(T_{h}\right)$ and cold heat baths $\left(T_{c}\right)$. More precisely, the relation was given as $\eta^{(2)} \equiv \frac{\left\langle W^{2}\right\rangle_{c}}{\left\langle Q^{2}\right\rangle_{c}} \leq \eta_{C}^{2}$, where $\eta_{C}=1-\frac{T_{c}}{T_{h}}$ is the Carnot efficiency and $\left\langle A^{2}\right\rangle_{c}=\left[\left\langle A^{2}\right\rangle-\langle A\rangle^{2}\right]$ is the second cumulant, or the fluctuations, of an observable $A$. Furthermore, a tighter-than-Carnot efficiency bound was derived for classical continuous engines expressed in terms of high order fluctuations of the power [36]. Our work exposes the relationship between these different bounds on fluctuations for general, quantum or classical continuous thermal machines, in linear response.

In this letter, we focus on generic steady-state thermal machines and prove the existence of lower and upper bounds for the ratio of output to input fluctuations, 
$\eta^{(2)}$. First, we prove that in the linear response regime $\langle\eta\rangle^{2} \leq \eta^{(2)} \leq \eta_{C}^{2}$, where $\langle\eta\rangle$ is the average efficiency of the engine with $\eta_{C}$ as the maximal average efficiency. These bounds follow from the Onsager reciprocity relation. Second, we uncover the relationship between $\eta^{(2)}$ and the TUR: For general multi-affinity systems operating as heat engines, the relative uncertainty of the output current is always greater than the relative uncertainty of the heat current absorbed by the engine from the hot bath. Third, we illustrate the validity of the $\eta^{(2)}$ bounds within the linear response regime for continuous-autonomous machines: thermoelectric junctions and quantum absorption refrigerators (QARs), depicted in Fig. 1. Furthermore, based on simulations, we suggest the validity of our bounds in a broader regime, beyond linear response thermodynamics.

Universal bounds in the linear response regime.- We consider a small-scale continuous engine: a system subjected to two thermodynamic forces (affinities) $A_{1}$ and $A_{2}$, which produce time-integrated stochastic currents $J_{i}$, $i=1,2$. The affinities (temperature, chemical potential) are properties of the reservoirs and as thermodynamic variables they negligibly fluctuate. In contrast, the currents may suffer from significant fluctuations. In steady state, typically, cumulants of integrated currents scale extensively with the operation time $t,\left\langle J_{i}^{n}\right\rangle_{c}=t\left\langle I_{i}^{n}\right\rangle_{c}$. In the linear response regime, the time-intensive average currents can be expressed in terms of the Onsager response matrix $[37,38]$ as $\left\langle I_{i}\right\rangle=\sum_{j=1,2} L_{i j} A_{j}$. The index $i=1,2$ corresponds to two different currents. $L_{i j}$ are the Onsager coefficients defined as $L_{i j}=\left.\partial_{A_{j}}\left\langle I_{i}\right\rangle\right|_{A=0}=L_{j i}$ for time-reversal systems. As per our convention, average currents $\left\langle I_{i}\right\rangle$ are positive when flowing towards the system. Considering now fluctuations of currents around their mean values, we define the squared relative uncertainty for individual currents as $\epsilon_{i}^{2}=\left\langle I_{i}^{2}\right\rangle_{c} /\left\langle I_{i}\right\rangle^{2}$ and construct the ratio between the uncertainties of the two currents in the linear response regime,

$$
\mathcal{Q} \equiv \frac{\epsilon_{2}^{2}}{\epsilon_{1}^{2}}=\frac{L_{22}}{L_{11}} \frac{\sum_{i j} L_{1 i} L_{1 j} A_{i} A_{j}}{\sum_{i j} L_{2 i} L_{2 j} A_{i} A_{j}} .
$$

Since the currents are linear in affinities, the fluctuation of currents are replaced by their corresponding equilibrium values in the linear response limit, $\left[\left\langle I_{i}^{2}\right\rangle_{c}\right]_{\mathrm{eq}}=L_{i i}$, ensuing from the fluctuation-dissipation relation in equilibrium. After simple algebraic manipulations, Eq. (1) reduces to

$$
\mathcal{Q}=1+\frac{1}{L_{11}\left\langle I_{2}\right\rangle^{2}} \sum_{i j}\left[L_{1 i} L_{1 j} L_{22}-L_{2 i} L_{2 j} L_{11}\right] A_{i} A_{j} .
$$

Interestingly, the above summation does not contribute for $i \neq j$ and therefore reduces to

$$
\mathcal{Q}=1+\frac{1}{L_{11}\left\langle I_{2}\right\rangle^{2}} \sum_{i}\left[L_{1 i}^{2} L_{22}-L_{2 i}^{2} L_{11}\right] A_{i}^{2} .
$$

The term in the sum can be written as a product of $\left(L_{22} A_{2}^{2}-L_{11} A_{1}^{2}\right)\left(L_{12}^{2}-L_{11} L_{22}\right)$, which in general can take an arbitrary sign. However, for time-reversal symmetric systems, due to the non-negativity property of the net entropy production rate in steady state, $\langle\sigma\rangle=$ $\sum_{i j}\left\langle I_{i}\right\rangle A_{i}=\sum_{i j} L_{i j} A_{i} A_{j} \geq 0$, Onsager's response coefficients satisfy the inequality $L_{12}^{2}-L_{11} L_{22} \leq 0$, thus the second term in the product above is always negative. So-far, we have not imposed any restriction on the operational behavior of the system. However, upon imposing the condition that the steady-state setup operates as an engine, it turns out the other term is also negative, $\left(L_{22} A_{2}^{2}-L_{11} A_{1}^{2}\right) \leq 0$. Proof: To realize a steady-state engine, we assign $I_{1}\left(I_{2}\right)$ as the input (output) channel. Recall that, as per our convention, the current flowing into the system is considered as positive. We therefore demand that $\left\langle I_{1}\right\rangle A_{1} \geq 0$ which in the linear response produces the condition $L_{11} A_{1}^{2} \geq-L_{12} A_{1} A_{2}$. Similarly, the system should deliver power i.e., $-\left\langle I_{2}\right\rangle A_{2} \geq 0$, which yields another condition, $-L_{12} A_{1} A_{2} \geq L_{22} A_{2}^{2}$. Combining these two conditions we receive the inequality

$$
L_{22} A_{2}^{2}-L_{11} A_{1}^{2} \leq 0
$$

which holds as long as the steady-state setup delivers power. Back to Eq. (3), we conclude that $\mathcal{Q} \geq 1$ in three operational regimes, see Table. This is the central result of our paper, and it allows us to conclude the following:

First, the condition $\mathcal{Q} \geq 1$ immediately implies that the TUR product $\left(\langle\sigma\rangle \frac{\left\langle I_{i}^{2}\right\rangle_{c}}{\left\langle I_{i}\right\rangle^{2}}\right)$ for the output current is always lower-bounded by the corresponding TUR product for the input current,

$$
\langle\sigma\rangle \frac{\left\langle I_{2}^{2}\right\rangle_{c}}{\left\langle I_{2}\right\rangle^{2}} \geq\langle\sigma\rangle \frac{\left\langle I_{1}^{2}\right\rangle_{c}}{\left\langle I_{1}\right\rangle^{2}} .
$$

Earlier studies [26, 27] derived independent TUR bounds for the individual currents for generic classes of steadystate systems (both Markovian and non-Markovian). In contrast, Eq. (5) shows that these bounds are not independent in the operational regime.

Second, an immediate consequence of $\mathcal{Q} \geq 1$ is that the ratio between fluctuations of currents gets lower-bounded by the square value of the averaged efficiency,

$$
\eta^{(2)}=\frac{A_{2}^{2}\left\langle I_{2}^{2}\right\rangle_{c}}{A_{1}^{2}\left\langle I_{1}^{2}\right\rangle_{c}} \geq\left[\frac{-A_{2}\left\langle I_{2}\right\rangle}{A_{1}\left\langle I_{1}\right\rangle}\right]^{2}=\langle\eta\rangle^{2},
$$

where $\langle\eta\rangle \equiv-A_{2}\left\langle I_{2}\right\rangle / A_{1}\left\langle I_{1}\right\rangle$ is the average efficiency of the machine.

Third, based on Eq. (4) we derive an upper bound for $\eta^{(2)}$ in the linear response regime. Using $L_{22}=\left\langle I_{2}^{2}\right\rangle_{c}$ and $L_{11}=\left\langle I_{1}^{2}\right\rangle_{c}$ we conclude that $\eta^{(2)}=\frac{A_{2}^{2} L_{22}}{A_{1}^{2} L_{11}} \leq 1$. Here, the unity on the right hand side corresponds to the square of the scaled Carnot efficiency, thus (see Table) for an engine we write the upper bound as

$$
\eta^{(2)} \leq \eta_{C}^{2}
$$


Altogether, we find that for an engine in linear response, $\langle\eta\rangle^{2} \leq \eta^{(2)} \leq \eta_{C}^{2}$, which is our main result. This inequality further provides a tighter bound on the average efficiency, $\langle\eta\rangle \leq \sqrt{\eta^{(2)}} \leq \eta_{C}$. This result applies for quantum or classical thermal machines, and in the Table we show the form of the bounds for engines, refrigerators and heat pumps. The bounds are simultaneously saturated in the reversible limit, when the output power [Eq. (4)] vanishes. Another interesting limit is when the input and the output currents are tightly coupled [16], resulting in a vanishing determinant of the Onsager response matrix, thus $\mathcal{Q}=1$. In this case, only the lower bound in (7) is saturated. Moreover, if the tight-coupling condition is satisfied for the stochastic currents, $I_{1} \propto I_{2}$, (and not only for the averaged currents, $\left\langle I_{1}\right\rangle \propto\left\langle I_{2}\right\rangle$ ), one receives $\mathcal{Q}=1$ even arbitrarily far from equilibrium, leading to $\eta^{(2)}=\langle\eta\rangle^{2} \leq \eta_{C}^{2}$. Additional bounds on ratios of high order cumulants in the tight coupling limit are presented in [39]. The special case, when a single affinity is applied (one of the $A_{i}=0$ ) is discussed in [39]. We now illustrate the bounds on $\eta^{(2)}$ for steady state autonomous thermal machines.

Example I: Thermoelectric transport in two-terminal systems.- We consider a two-terminal thermoelectric device depicted in Fig. 1(a). The junction consists of an elastic scatter (e.g., array of quantum dots), which is connected to two fermionic reservoirs. For such a noninteracting setup the full-counting statistics for both charge and heat currents can be obtained exactly using a scattering matrix formalism. It is given by the celebrated Levitov-Lesovik formula [40-42]. Expressions of the currents and their higher order cumulants are given in [39]. $\mu_{L, R}$ and $T_{L, R}$ denote the electrochemical potential and temperatures of the left $(L)$ and right $(R)$ electronic reservoirs, $\Delta T=T_{R}-T_{L}$, with $T=\left(T_{L}+T_{R}\right) / 2$ the average temperature. To realize different operational regimes, we set the thermodynamic parameters for the reservoirs as $T_{R}>T_{L}$ and $\mu_{L}>\mu_{R}$. In a thermoelectric engine, the heat current absorbed from the hot (right) terminal is used to drive charge current against the chemical bias. The thermodynamic affinities responsible for realizing an engine are $A_{1}=\eta_{C} / T_{L}$ and $A_{2}=1 / T_{L}$, see Table. When the system operates as a refrigerator or a heat pump, the (left) cold terminal is cooled down using electrochemical work. Following consistent stochastic thermodynamics framework [16], the stochastic output power for the setup is written as $-\dot{w} \equiv\left(\mu_{L}-\mu_{R}\right) I_{e}^{R}$ which on average is positive (negative) for engine (refrigerator). Correspondingly, the stochastic heat current is given as $\dot{q}_{\alpha} \equiv I_{q}^{\alpha}=I_{u}^{\alpha}-\mu_{\alpha} I_{e}^{\alpha}, \alpha=L, R$. Currents here are defined positive when entering the system from the right terminal.

In Fig. 2 we display contour plots of (a)-(b) $\eta^{(2)}-\langle\eta\rangle^{2}$ and (c)-(d) $\eta_{C}^{2}-\eta^{(2)}$ for a thermoelectric engine both in linear response (LR) and far from equilibrium (FFE). We use a single quantum dot as the scatter with the dot energy $\epsilon_{d}$ and the hybridization strength $\Gamma$, which is assumed to be equal for both left and right leads. These simulations exemplify the validity of the bounds. To further establish the lower and upper bounds for an engine beyond the linear response regime, we employ the following numerical procedure: Beginning with a point in our parameter space $\left(\Gamma\right.$ and $\epsilon_{d}$ ), and selecting particular biases $\Delta \mu$ and $\Delta T, \eta^{(2)}$ takes a certain value, generally away from the bounds. Focusing on the lower bound (and similarly for the upper bound), we move in parameter space according to a stochastic optimization algorithm (ADAM [43]), by minimizing a cost function, which is a function of $\eta^{(2)}-\langle\eta\rangle^{2}$ (see [39] for details). This process is performed for a large ensemble of around 1100 initial conditions, near and inside the engine domain. In Fig. 3, we show the resulting distribution of the minima $\eta^{(2)}-\langle\eta\rangle^{2}$ and $\eta_{C}^{2}-\eta^{(2)}$. We begin with a uniform sample of points inside the engine regime. We then present histograms after 1000 and 3000 optimization steps. As the number of steps increases, more points are nearing saturation of the bound as expected for the optimization process. In the insets we illustrate that for each step the differences above are positive throughout the optimization process by plotting four random minimization paths, along with the average of all paths. This powerful procedure provides numerical evidence for the validity of the bounds for the single dot engine beyond linear response. Furthermore, it reveals that the lower bound is saturated in the tight coupling limit, as values for $\eta^{(2)}-\langle\eta\rangle^{2}$ after minimization correspond to diminishing $\Gamma$.

We complement these results in [39] with (i) simulations demonstrating that analogous bounds for $\eta^{(2)}$ are satisfied for single-dot refrigerators and pumps in linear response, along with examples suggesting the validity of the bounds in the FFE regime. (ii) Simulations of thermoelectric engines based on a serial double-dot system. Here, we show that the lower and upper bounds for $\eta^{(2)}$ hold beyond linear response, even when the standard Markovian TUR $\left(\langle\sigma\rangle \frac{\left\langle I_{i}^{2}\right\rangle_{c}}{\left\langle I_{i}\right\rangle^{2}} \geq 2\right)$ is violated.

Example II: Quantum Absorption Refrigerators. Models for QARs have served a crucial role in establishing working principles of autonomous quantum thermal machines [44-46] with recent experiments realizing such models using trapped ions $[8,9]$. In QARs, heat is extracted from a cold $(c)$ bath and released into a hot $(h)$ environment by utilizing heat from a so-called work $(w)$ bath. The reversed operation realizes a heat engine. We identify three temperatures, $T_{w}>T_{h}>T_{c}$ in a QAR, and three heat currents, $I_{c, h, w}$, defined positive when flowing towards the system. Schematic diagrams of three-level and four-level QARs are displayed in Figs. 4(a) and 4(b), respectively. In what follows, we limit our discussion to the weak system-bath coupling limit, which can be handled with perturbative quantum Master equations. While so-far most studies characterized QARs 
TABLE I. Input $\left(I_{1}\right)$ and output $\left(I_{2}\right)$ currents, their affinities, average efficiency and ratio of fluctuations in different operational regimes following the notation of Ref. [19]. The delivered power is given by $-I_{2} . \eta_{C}=1-T_{c} / T_{h}$ is the Carnot efficiency.

\begin{tabular}{cccc}
\hline \hline & Heat Engine & Refrigerator & Heat Pump \\
\hline$I_{1}$ & $\dot{q}_{h}$ & $\dot{w}$ & $\dot{w}$ \\
$A_{1}$ & $\eta_{C} / T_{c}$ & $1 / T_{h}$ & $1 / T_{c}$ \\
$I_{2}$ & $\dot{w}$ & $-\dot{q}_{c}$ & $\dot{q}_{h}$ \\
$A_{2}$ & $1 / T_{c}$ & $\eta_{C} / T_{c}$ & $\eta_{C} / T_{c}$ \\
$\langle\eta\rangle$ & $\frac{-\langle\dot{w}\rangle}{\left\langle\dot{q}_{h}\right\rangle} \leq \eta_{C}$ & $\frac{\left\langle\dot{q}_{c}\right\rangle}{\langle\dot{w}\rangle} \leq \frac{\left(1-\eta_{C}\right)}{\eta_{C}}$ & $\frac{\left\langle-\dot{q}_{h}\right\rangle}{\langle\dot{w}\rangle} \leq \frac{1}{\eta_{C}}$ \\
$\eta^{(2)}$ & $\langle\eta\rangle_{\mathrm{eng}}^{2} \leq \eta_{\mathrm{eng}}^{(2)} \leq \eta_{C}^{2}$ & $\langle\eta\rangle_{\mathrm{ref}}^{2} \leq \eta_{\mathrm{ref}}^{(2)} \leq\left(\frac{1-\eta_{C}}{\eta_{C}}\right)^{2}$ & $\langle\eta\rangle_{\mathrm{pump}}^{2} \leq \eta_{\mathrm{pump}}^{(2)} \leq \frac{1}{\eta_{C}^{2}}$ \\
\hline
\end{tabular}
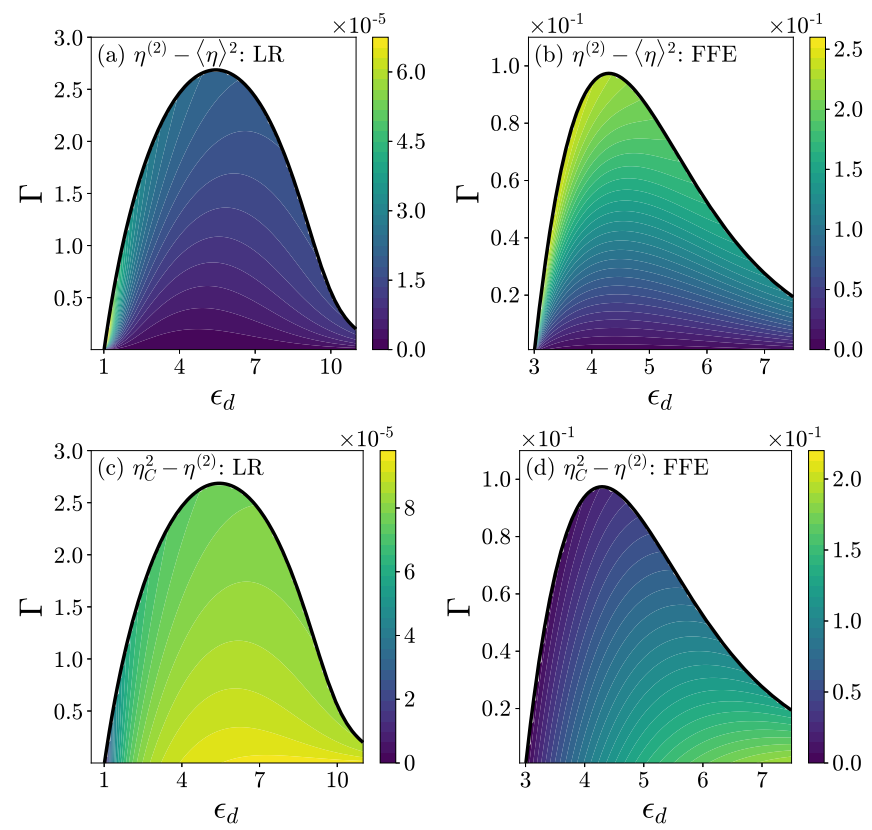

FIG. 2. (Color online) (a)-(b) Test of the lower bound, $\eta^{(2)} \geq$ $\langle\eta\rangle^{2}$, and (c)-(d) the upper bound, $\eta_{C}^{2} \geq \eta^{(2)}$, for a singledot thermoelectric system within linear response (LR) and far from equilibrium (FFE). We focus on the ranges of $\epsilon_{d}$ and $\Gamma$ at which the system operates as an engine. Positive values signify that bounds are satisfied (see Table I). We use $\beta_{L}=1.01, \beta_{R}=1, \mu_{L}=0.01, \mu_{R}=0$ for (a) and (c) and $\beta_{L}=2, \beta_{R}=1, \mu_{L}=1, \mu_{R}=-1$ for (b) and (d).

based on their averaged currents [47], fluctuations of currents in QARs were analyzed recently in Refs. [48, 49], demonstrating e.g. the nontrivial impact of quantum coherences on the cooling current and its fluctuation.

The cooling efficiency of QARs is defined as $\langle\eta\rangle=$ $\left\langle I_{c}\right\rangle /\left\langle I_{w}\right\rangle$. It is bounded by $\langle\eta\rangle \leq \eta_{\text {cool }}$ where $\eta_{\text {cool }} \equiv$ $\frac{\left(\beta_{h}-\beta_{w}\right)}{\beta_{c}-\beta_{h}} \stackrel{T_{w} \gg T_{h}}{\longrightarrow}\left(1-\eta_{C}\right) / \eta_{C}[45]$. For a three-level QAR with energy levels sketched in Fig. 4(a), we get $\langle\eta\rangle=\theta_{c} / \theta_{w}$. Here, $\theta_{c, w}$ are energy gaps with transitions activated by the cold and work baths, respectively. Furthermore, based on the formalism of the truncated cumulant generating function [48], we obtain here the fluctuations of the currents, $\eta^{(2)} \equiv \frac{\left\langle I_{c}^{2}\right\rangle_{c}}{\left\langle I_{w}^{2}\right\rangle_{c}}=\left(\frac{\theta_{c}}{\theta_{w}}\right)^{2}$. We therefore
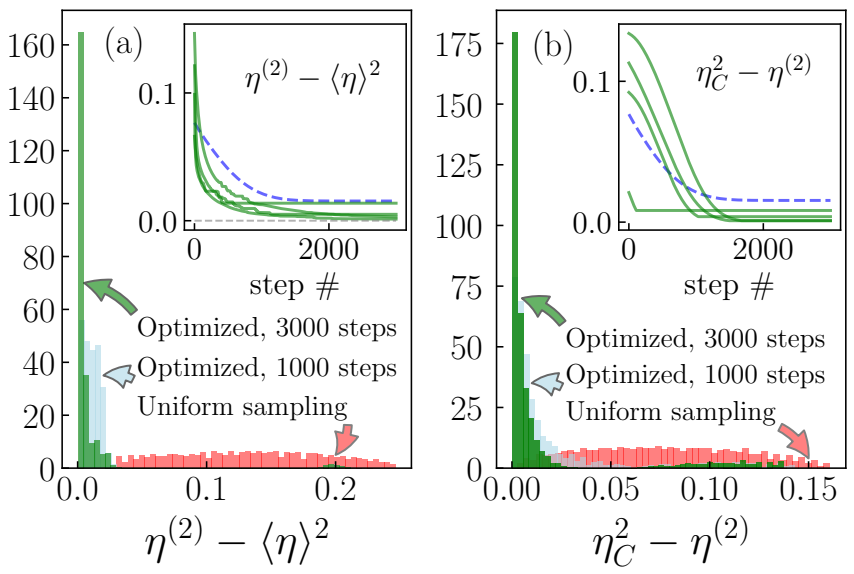

FIG. 3. (Color online) Distributions of the difference (a) $\eta^{(2)}-\langle\eta\rangle^{2}$ and (b) $\eta_{C}^{2}-\eta^{(2)}$ for a single-dot thermoelectric junction beyond linear response. The three cases shown are of a uniform sampling (red) of the engine region (see Fig. 2 (b) and (d)), and the result of a stochastic optimization of 1000 steps (light blue) and 3000 steps (green). We do not observe a violation of the bound throughout the minimization process. The insets show the mean value of the optimization path (in dashed blue), and samples of optimization paths for random initial points in parameter space (green). We use the same parameters as in Fig. 2(b).

conclude that the three-level QAR satisfies at weak coupling $\langle\eta\rangle^{2}=\eta^{(2)} \leq \eta_{\text {cool }}^{2}$. The upper limit is deduced based on our analysis of linear response. The saturation of the lower bound holds even in the far-from-equilibrium regime, and it corresponds to the tight-coupling limit, which holds under the weak coupling approximation.

To explore behavior beyond the tight-coupling limit, we sketch in Fig. 4(b) a four-level QAR with nondegenerate intermediate levels. At weak coupling, the two cooling cycles compete. As a result, the maximal efficiency $\eta_{\text {cool }}$ cannot be achieved and the system always dissipates heat [50]. We simulate fluctuations of the currents in the incoherent limit [39,48], and exemplify the behavior of this model in Fig. 4(c). As expected, deviations from the tight-coupling limit become more pronounced as we increase the gap between the intermediate levels. The extreme case $g \ll \gamma \theta_{c, w}$ is presented to illustrate the saturation of the bound in the tight coupling 

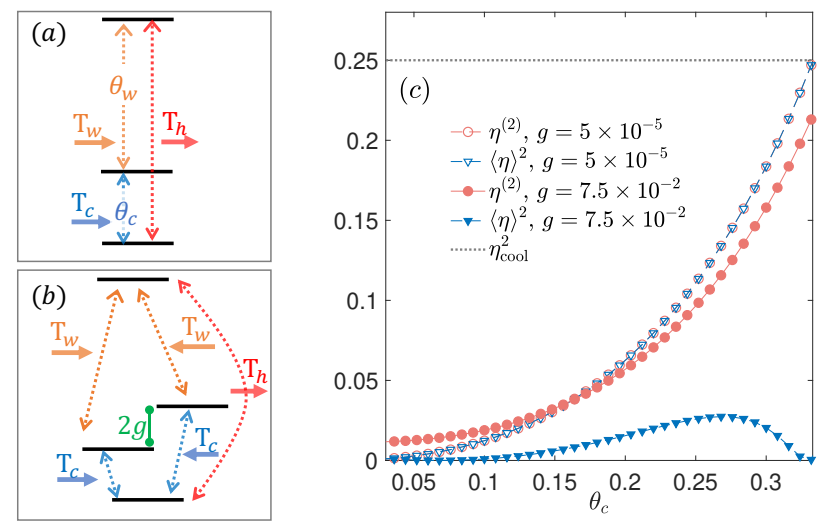

FIG. 4. (Color online) (a) Three-level and (b) four-level models for QARs. Dashed arrows represent bath-induced transitions. Full-line arrows show the direction of heat absorbed and released when the system operates as a QAR. (c) Simulations of $\eta^{(2)}$ for the four-level model (b). Here, $\theta_{c}$ represents the energy gap between the ground level to halfway between the intermediate levels. We use $T_{w}=0.4$, $T_{h}=0.2, T_{c}=0.1$, with the maximum cooling efficiency $\eta_{\text {cool }} \equiv \frac{\beta_{h}-\beta_{w}}{\beta_{c}-\beta_{h}}=0.5$. The spectral function of the baths are Ohmic, $J_{b}(\omega)=\gamma_{b} \omega e^{-|\omega| / \Lambda}$, with $\gamma_{h}=2 \times 10^{-3}, \gamma_{w, c}=10^{-3}$, $\Lambda=50$.

limit, $\eta^{(2)}=\langle\eta\rangle^{2}$, further achieving the upper bound $\eta_{\text {cool }}^{2}$ at a certain value. As we increase the gap, $g$, the two cycles compete, the tight-coupling limit no longer holds, and we observe nontrivial results: Both lower and upper bounds for $\eta^{(2)}$ are satisfied - even beyond the linear response regime. We further confirmed with simulations the validity of these bounds in broad range of parameters $g, \gamma$ and $\theta_{c, w}$.

Perspective.- Beyond the averaged behavior of observables, the performance of small thermal machines should be characterized by their fluctuations. In this work, we proved that in linear response, relative fluctuations of the output current (power in heat engines) are greater than fluctuations in the input current (absorbed heat) in the thermal engine regime. We immediately translated this inequality into universal lower and upper bounds on $\eta^{(2)}$, the ratio between the fluctuations of output and input channels, which hold in linear response thermodynamics. Numerical simulations of quantum dots thermoelectric junctions and quantum absorption refrigerators suggest the broader validity of these bounds. Proofs of bounds on fluctuations and higher order cumulants beyond linear response for general (possibly quantum) dynamics remain as an open challenge.

BKA acknowledges the MATRICS grant MTR/2020/000472 from SERB, Government of India. BKA and DS thank the Shastri Indo-Canadian Institute for providing financial support for this research work in the form of a Shastri Institutional Collaborative Research Grant (SICRG). DS acknowledges support from an NSERC Discovery Grant and the Canada Research Chair program. The work of IK was supported by the CQIQC at the University of Toronto. The research of MG was funded by an NSERC Graduate Scholarship - Master's (CGS M). SS acknowledge support from the Council of Scientific \& Industrial Research (CSIR), India (Grant Number 1061651988).

[1] H. B. Callen, Thermodynamics and an Introduction to Thermostatistics, 2nd edition 1985, Wiley, New York.

[2] E. H. Lieb, J. Yngvason, The Physics and Mathematics of the Second Law of Thermodynamics, Phys. Rept. 310 1 (1999).

[3] J. Klatzow et. al., Experimental Demonstration of Quantum Effects in the operation of Microscopic Heat Engines, Phys. Rev. Lett. 122, 110601 (2019).

[4] R. Uzdin, A. Levy, and R. Kosloff, Equivalence of quantum heat machines, and quantum-thermodynamic signatures, Phys. Rev. X 5, 031044 (2015).

[5] S. Krishnamurthy, S. Ghosh, D. Chatterji, R. Ganapathy, and A. K. Sood, A micrometre-sized heat engine operating between bacterial reservoirs, Nat. Phys. 12, 1134 (2016).

[6] J. P. S. Peterson, T. B. Batalhão, M. Herrera, A. M. Souza, R. S. Sarthour, I. S. Oliveira, and R. M. Serra, Experimental Characterization of a Spin Quantum Heat Engine, Phys. Rev. Lett 123, 240601 (2019).

[7] D. von Lindenfels, O. Gräb, C. T. Schmiegelow, V. Kaushal, J. Schulz, M. T. Mitchison, J. Goold, F. Schmidt-Kaler, and U. G. Poschinger, Spin heat engine coupled to a harmonic-oscillator flywheel, Phys. Rev. Lett. 123, 080602 (2019).

[8] J. Klatzow, J. N. Becker, P. M. Ledingham, C. Weinzetl, K. T. Kaczmarek, D. J. Saunders, J. Nunn, I. A. Walmsley, R. Uzdin, and E. Poem, Quantum absorption refrigerator with trapped ions, Phys. Rev. Lett. 122, 110601 (2019).

[9] G. Maslennikov, et al., Quantum absorption refrigerator with trapped ions, Nat. Comm. 10, 202 (2019).

[10] R. Kubo, The fluctuation-dissipation theorem, Rep. Prog. Phys. 29, 255 (1966).

[11] U. M. B. Marconi, A. Puglisi, L. Rondoni, and A. Vulpiani, Fluctuation-dissipation: Response theory in statistical physics, Phys. Rep. 461, 111 (2008).

[12] M. Baiesi, C. Maes, and B. Wynants, Fluctuations and Response of Nonequilibrium States, Phys. Rev. Lett. 103, 010602 (2009).

[13] R. Kosloff, Quantum thermodynamics and open-systems modeling, J. Chem. Phys. 150, 204105 (2019).

[14] U. Seifert, Stochastic thermodynamics, fluctuation theorems, and molecular machines, Rep. Prog. Phys. 75, 126001 (2012).

[15] U. Seifert, Stochastic thermodynamics: Principles and perspective, Eur. Phys. J. B 64, 423 (2008).

[16] C. V. den Broeck, Stochastic thermodynamics: A brief introduction, in Physics of Complex Colloids, edited by C. Bechinger, F. Sciortino, and P. Ziherl (IOS Press, Amsterdam, 2013), pp. 155-194.

[17] S. Vinjanampathy and J. Anders, Quantum thermody- 
namics, Contemporary Physics 57, 545 (2016).

[18] R. Kosloff, Quantum Thermodynamics: A Dynamical Viewpoint, Entropy 15, 2100 (2013).

[19] G. Verley, T. Willaert, C. Van den Broeck and M. Esposito, Universal theory of efficiency fluctuations, Phys. Rev. E 90, 052145 (2014).

[20] G. Verley, M. Esposito, T. Willaert and C. Van den Broeck, The unlikely Carnot efficiency, Nature Communications 5, 4721 (2014)

[21] O. S. Lumbroso, L. Simine, A. Nitzan, D. Segal, and O. Tal Electronic noise due to temperature differences in atomic-scale junctions, Nature 562, 240 (2018).

[22] S. Yuan, T. Gao, W. Cao, Z. Pan, J. Liu, J. Shi, and W. Hong, The Characterization of Electronic Noise in the Charge Transport through Single-Molecule Junctions, Small Methods 5, 2001064 (2021).

[23] M. Esposito, U. Harbola, and S. Mukamel, Nonequilibrium fluctuations, fluctuation theorems, and counting statistics in quantum systems, Rev. Mod. Phys. 81, 1665 (2009).

[24] M. Campisi, P. Hänggi, and P. Talkner, Quantum fluctuation relations: Foundations and applications, Rev. Mod. Phys. 83, 771 (2011).

[25] C. Jarzynski, Equalities and Inequalities: Irreversibility and the Second Law of Thermodynamics at the Nanoscale, Ann. Rev. Cond. Mat. Phys. 2, 329 (2011).

[26] A. C. Barato and U. Seifert, Thermodynamic uncertainty relation for biomolecular processes, Phys. Rev. Lett. 114, 158101 (2015).

[27] P. Pietzonka and Udo Seifert, Universal Trade-Off between Power, Efficiency, and Constancy in Steady-State Heat Engines, Phys. Rev. Lett. 120, 190602 (2018).

[28] T. R. Gingrich, J. M. Horowitz, N. Perunov, and J. L. England, Dissipation bounds all steady state current fluctuations, Phys. Rev. Lett. 116, 120601 (2016).

[29] G. Falasco, M. Esposito, and J.-C. Delvenne, Unifying thermodynamic uncertainty relations, New J. Phys. 22, 053046 (2020).

[30] K. Macieszczak, K. Brandner, and J. P. Garrahan, Unified thermodynamic uncertainty relations in linear response, Phys. Rev. Lett. 121, 130601 (2018).

[31] A. M. Timpanaro, G. Guarnieri, J. Goold, and G. T. Landi, Thermodynamic uncertainty relations from exchange fluctuation theorems, Phys. Rev. Lett. 123, 090604 (2019).

[32] K. Brandner, T. Hanazato, and K. Saito, Thermodynamic bounds on precision in ballistic multiterminal transport, Phys. Rev. Lett. 120, 090601 (2018).

[33] J. Liu and D. Segal, Thermodynamic uncertainty relation in quantum thermoelectric junctions, Phys. Rev. E 99, 062141 (2019).

[34] S. Saryal, O. Sadekar, and B. K. Agarwalla, Thermodynamic uncertainty relation for energy transport in a transient regime: A model study, Phys. Rev. E 103, 022141 (2021).

[35] K. Ito, C. Jiang, and G. Watanabe, Universal Bounds for Fluctuations in Small Heat Engines, arXiv:1910.08096.

[36] T. Kamijima, S. Otsubo, Y. Ashida, and T. Sagawa, Higher order efficiency bound and its application to nonlinear nano-thermoelectrics, arXiv:2103:06554.

[37] G. Benenti, G. Casati, K. Saito, and R. Whitney, Fundamental aspects of steady-state conversion of heat to work at the nanoscale, Phys. Rep. 694, 1 (2017).

[38] J. H. Jiang, B. K. Agarwalla, and D. Segal, Efficiency
Statistics and Bounds for Systems with Broken TimeReversal Symmetry, Phys. Rev. Lett. 115, 040601 (2015). [39] Supplemental Material

[40] L. S. Levitov and G. B. Lesovik, Charge distribution in quantum shot noise, Pis'ma Zh. Eksp. Teor. Fiz. 58, 225 (1993) [JETP Lett. 58, 230 (1993)].

[41] K. Schönhammer, Full counting statistics for noninteracting fermions: Exact results and the Levitov-Lesovik formula, Phys. Rev. B 75, 205329 (2007).

[42] B. K. Agarwalla, B. Li, and J.-S. Wang, Full-counting statistics of heat transport in harmonic junctions: Transient, steady states, and fluctuation theorems, Phys. Rev. E 85, 051142 (2012).

[43] Diederik P. Kingma and Jimmy Ba, Adam: A method for stochastic optimization, arXiv:1412.6980.

[44] R. Kosloff and A. Levy, Quantum heat engines and refrigerators: Continuous devices, Annu. Rev. Phys. Chem. 65, 365 (2014).

[45] L. A. Correa, J. P. Palao, D. Alonso, and G. Adesso, Quantum-enhanced absorption refrigerators, Scientific reports 4, 1 (2014).

[46] M. T. Mitchison, Quantum thermal absorption machines: refrigerators, engines and clocks, Contemporary Physics 60, 164 (2019).

[47] M. Kilgour and D. Segal, Coherence and decoherence in quantum absorption refrigerators, Phys. Rev. E 98, 012117 (2018).

[48] D. Segal, Current fluctuations in quantum absorption refrigerators, Phys. Rev. E 97, 052145 (2018).

[49] J. Liu and D. Segal, Coherences and the thermodynamic uncertainty relation: Insights from quantum absorption refrigerators, Phys. Rev. E 103, 032138 (2021).

[50] L. A. Correa, J. P. Palao, and D. Alonso, Internal dissipation and heat leaks in quantum thermodynamic cycles, Phys. Rev. E 92, 032136 (2015). 


\section{Supplemental Material: Universal Bounds on Fluctuations in Continuous Thermal Machines}

\section{THERMOELECTRIC TRANSPORT IN NONINTERACTING QUANTUM JUNCTIONS}

\section{Expressions for currents and noise from full counting statistics}

In this section, we provide expressions for cumulants or charge and energy currents for generic quantum noninteracting thermoelectric junction. This setup is considered in the main text to illustrate our main findings. The exact cumulant generating function (CGF) for this problem was obtained before with a scattering matrix approach. It is given by the celebrated Levitov-Lesovik formula [40-42]. The CGF is defined from the characteristic function $\mathcal{Z}\left(\chi_{e}, \chi_{u}\right)=\left\langle\exp \left(i \chi_{e} n_{e}+i \chi_{u} h_{u}\right)\right\rangle$, where $\chi_{e}$ and $\chi_{u}$ are the counting fields that keep track of net charge $n_{e}$ and energy transfer $h_{u}$ processes, respectively, in the right terminal. In the long-time limit, the CGF scales extensively with the integrated time $t$. The corresponding (scaled) cumulant generating function (CGF), i.e., $G\left(\chi_{e}, \chi_{u}\right)=\lim _{t \rightarrow \infty} \frac{1}{t} \ln Z\left(\chi_{e}, \chi_{u}\right)$, is given as,

$$
\begin{aligned}
& G\left(\chi_{e}, \chi_{u}\right)=\int_{-\infty}^{\infty} \frac{d \epsilon}{2 \pi} \ln \left(1+\mathcal{T}(\epsilon)\left\{f_{R}(\epsilon)\left[1-f_{L}(\epsilon)\right]\right.\right. \\
& \left.\left.\left[e^{i\left(\chi_{e}+\epsilon \chi_{u}\right)}-1\right]+f_{L}(\epsilon)\left[1-f_{R}(\epsilon)\right]\left[e^{-i\left(\chi_{e}+\epsilon \chi_{u}\right)}-1\right]\right\}\right) .
\end{aligned}
$$

As per our convention, currents flowing out of the right reservoir is considered positive. Here, $\mathcal{T}(\epsilon)$ is the transmission function, which indicates the probability for electrons to transfer from the right to the left reservoir via the elastic scattering region. $f_{\alpha}(\epsilon)=\left[e^{\beta_{\alpha}\left(\epsilon-\mu_{\alpha}\right)}+1\right]^{-1}, \alpha=L, R$ is the equilibrium Fermi-Dirac distribution function for the $\alpha$-th reservoir. From the CGF, we obtain cumulants of currents by taking derivatives with respect to the corresponding counting fields, and obtain

$$
\left\langle I_{K}\right\rangle=\int_{-\infty}^{\infty} \frac{d \epsilon}{2 \pi} \xi_{K} \mathcal{T}(\epsilon)\left[f_{R}(\epsilon)-f_{L}(\epsilon)\right]
$$

where $\xi_{K}=1(\epsilon)$ for $K=e(u)$. Fluctuations i.e., the second order cumulants are given by,

$$
\begin{aligned}
\left\langle I_{K}^{2}\right\rangle_{c}= & \int_{-\infty}^{\infty} \frac{d \epsilon}{2 \pi} \xi_{\alpha}^{2} \mathcal{T}(\epsilon)\left\{f_{L}(\epsilon)\left[1-f_{R}(\epsilon)\right]\right. \\
& \left.+f_{R}(\epsilon)\left[1-f_{L}(\epsilon)\right]\right\}-\mathcal{T}^{2}(\epsilon)\left[f_{R}(\epsilon)-f_{L}(\epsilon)\right]^{2} .
\end{aligned}
$$

Note that the heat current $(q)$ cumulants can be simply obtained by setting $\xi_{q}=\epsilon-\mu_{R}$. In the linear response regime, one considers the limit $|\Delta T| \ll T$ and $|\Delta \mu| \ll T$. Here, $\Delta \mu=\mu_{L}-\mu_{R}, \Delta T=T_{R}-T_{L}$, and $T=\left(T_{L}+T_{R}\right) / 2$, $\mu=\left(\mu_{L}+\mu_{R}\right) / 2$ being the average temperature and average chemical potential, respectively. The currents can then be expressed in terms of Onsager's transport coefficients,

$$
\begin{aligned}
& \left\langle I_{e}\right\rangle=L_{e e}\left(\frac{-\Delta \mu}{T}\right)+L_{e q}\left(\frac{\Delta T}{T^{2}}\right) \\
& \left\langle I_{q}\right\rangle=L_{q e}\left(\frac{-\Delta \mu}{T}\right)+L_{q q}\left(\frac{\Delta T}{T^{2}}\right),
\end{aligned}
$$

where we used $-\frac{\partial f}{\partial \epsilon}=f(1-f) \beta$. Here, $f(\epsilon)$ is the equilibrium distribution function evaluated at $T$ and $\mu$. The various transport coefficients are given by

$$
\begin{aligned}
L_{e e} & =\int_{-\infty}^{\infty} \frac{d \epsilon}{2 \pi} \mathcal{T}(\epsilon) f(\epsilon)[1-f(\epsilon)] \\
L_{e q} & =L_{q e}=\int_{-\infty}^{\infty} \frac{d \epsilon}{2 \pi}(\epsilon-\mu) \mathcal{T}(\epsilon) f(\epsilon)[1-f(\epsilon)] \\
L_{q q} & =\int_{-\infty}^{\infty} \frac{d \epsilon}{2 \pi}(\epsilon-\mu)^{2} \mathcal{T}(\epsilon) f(\epsilon)[1-f(\epsilon)]
\end{aligned}
$$



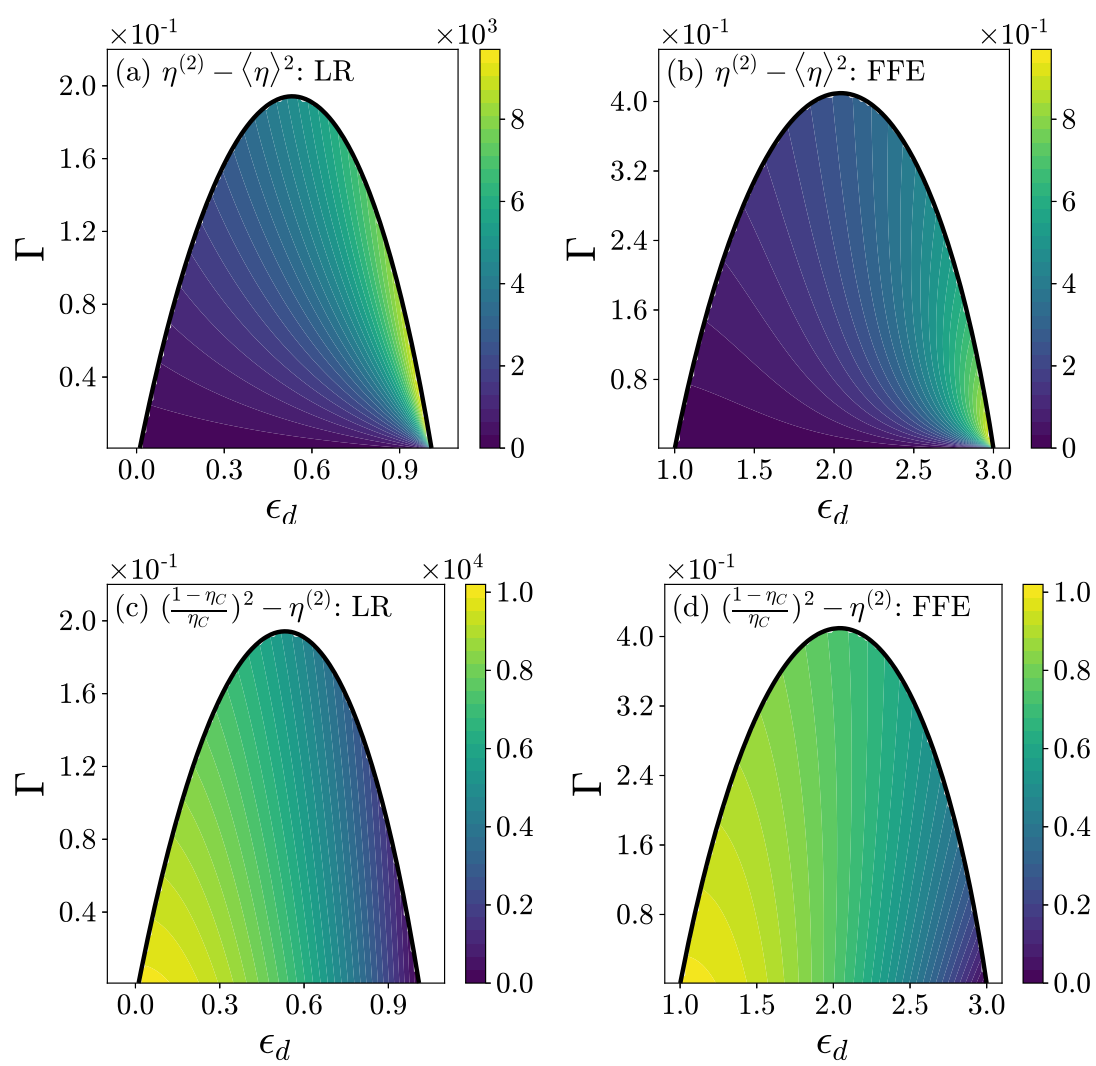

FIG. S1. (Color online) Single-dot thermoelectric refrigerator. (a)-(b) Test of the lower bound, $\eta^{(2)} \geq\langle\eta\rangle^{2}$ within linear response and far from equilibrium. (c)-(d) Test of the upper bound, $\left(\frac{1-\eta_{C}}{\eta_{C}}\right)^{2} \geq \eta^{(2)}$, for the same system. We focus on relevant ranges of $\epsilon_{d}$ and $\Gamma$ at which the system operates as a refrigerator. Positive values signify that the bound is satisfied (see Table in main text). We used $\beta_{L}=1.01, \beta_{R}=1, \mu_{L}=0.01, \mu_{R}=0$ for (a) and (c) and $\beta_{L}=2, \beta_{R}=1, \mu_{L}=1, \mu_{R}=-1$ for (b) and (d).

In the equilibrium limit $(\Delta \mu=\Delta T=0)$, one recovers the standard equilibrium fluctuation-dissipation relation from Eq. (S3),

$$
\left[\left\langle I_{q}^{2}\right\rangle_{c}\right]_{\mathrm{eq}}=L_{q q}, \quad\left[\left\langle I_{e}^{2}\right\rangle_{c}\right]_{\mathrm{eq}}=L_{e e}
$$

We now discuss three operational regimes for such a setup by setting $T_{R}>T_{L}$ and $\mu_{L}>\mu_{R}$.

1. Thermoelectric engine: In order to operate the setup as a thermoelectric engine, one must ensures that the net heat current absorbed from the hot reservoir $(R)$ is positive i.e., $\left\langle I_{u}\right\rangle-\mu_{R}\left\langle I_{e}\right\rangle \geq 0$. The heat absorbed leads to transport of charge current against the chemical bias, thus delivering power i.e., $-\langle\dot{w}\rangle=\left(\mu_{L}-\mu_{R}\right)\left\langle I_{e}\right\rangle \geq 0$. The corresponding efficiency is then $\langle\eta\rangle_{\text {eng }}=\frac{\left(\mu_{L}-\mu_{R}\right)\left\langle I_{e}\right\rangle}{\left\langle I_{u}\right\rangle-\mu_{R}\left\langle I_{e}\right\rangle}$.

2. Refrigerator: In the refrigerator regime, heat current from the cold (left) reservoir is extracted by using the charge current flowing from high to low bias i.e., $\left\langle I_{u}\right\rangle-\mu_{L}\left\langle I_{e}\right\rangle \leq 0$ and $\left\langle I_{e}\right\rangle \leq 0$. The efficiency is given as $\langle\eta\rangle_{\mathrm{ref}}=$ $\frac{-\left(\left\langle I_{u}\right\rangle-\mu_{L}\left\langle I_{e}\right)\right\rangle}{-\left(\mu_{L}-\mu_{R}\right)\left\langle I_{e}\right\rangle}$.

3. Heat pump: To realize a heat pump, we demand that the heat current should flow towards the (right) hot reservoir, $\left\langle I_{u}\right\rangle-\mu_{R}\left\langle I_{e}\right\rangle \leq 0$, by using the charge current, $\left\langle I_{e}\right\rangle \leq 0$. The corresponding efficiency is $\langle\eta\rangle_{\text {pump }}=\frac{-\left(\left\langle I_{u}\right\rangle-\mu_{R}\left\langle I_{e}\right\rangle\right)}{-\left(\mu_{L}-\mu_{R}\right)\left\langle I_{e}\right\rangle}$. 

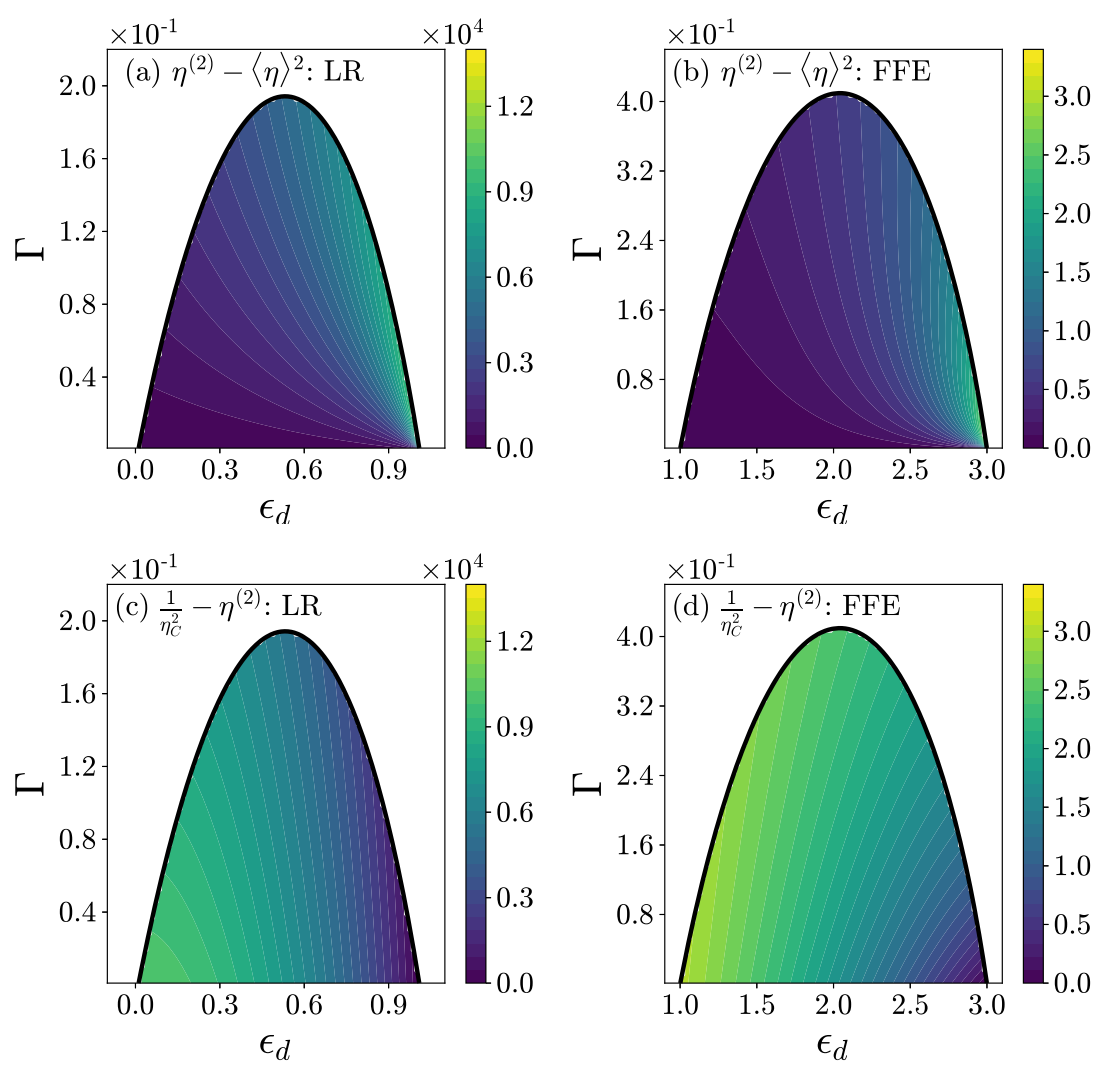

FIG. S2. (Color online) Single-dot thermoelectric heat pump. (a)-(b) Test of the lower bound, $\eta^{(2)} \geq\langle\eta\rangle^{2}$ within linear response and far from equilibrium. (c)-(d) Test of the upper bound, $\frac{1}{\eta_{C}^{2}} \geq \eta^{(2)}$, for the same system. We focus on relevant ranges of $\epsilon_{d}$ and $\Gamma$ at which the system operates as a heat pump. Positive values signify that the bound is satisfied (see Table in main text). We used $\beta_{L}=1.01, \beta_{R}=1, \mu_{L}=0.01, \mu_{R}=0$ for (a) and (c) and $\beta_{L}=2, \beta_{R}=1, \mu_{L}=1, \mu_{R}=-1$ for (b) and (d).

In the main text, we illustrate the validity of the lower and upper bounds for $\eta^{(2)}$ for a thermoelectric engine, within the linear response (LR) regime and far from equilibrium (FFE). Here, in Fig. S1 we display the validity for the corresponding lower, $\langle\eta\rangle^{2} \leq \eta^{(2)}$, and upper, $\eta^{(2)} \leq\left(\frac{1-\eta_{C}}{\eta_{C}}\right)^{2}$, bounds for a single-dot thermoelectric system in the refrigerator regime. Figure S2 presents analogous results for single-dot heat pumps.

In Fig. S3, we show the $\mathcal{Q}$ ratio in the same regime as displayed in Figs. S1-S2 both in the linear and beyond linear response regime. We observe the existence of the lower bound i.e., $\mathcal{Q} \geq 1$ in both the engine (E) and the refrigerator (R) regimes.

\section{Tight-coupling limit beyond linear response}

In this subsection we focus on the tight coupling (TC) limit. We prove the upper bound $\eta^{(2)} \leq \eta_{C}^{2}$ and that the lower bound is saturated. Both results hold beyond linear response. We further derive bounds on ratios (output power to heat) of high order cumulants.

In the tight-coupling limit, the energy current flowing through the junction is proportional to the electron flux. While commonly, this limit is assumed for the averaged currents, here we enforce it for the stochastic currents. i.e., $I_{u}=\epsilon_{d} I_{e}$ with $\epsilon_{d}$ the energy of the single quantum dot. Since the currents here are proportional (coupled), this limit is commonly referred to as the "tight-coupling limit". Such a situation can be easily realized when a single quantum level is weakly coupled to the reservoirs. The dynamics and thermodynamics of the model can be obtained from stochastic thermodynamics using Markovian master equations. The TC limit is of great interest in nanoelectronics since the system in this limit has the ability to operate at the Carnot efficiency [1]. Furthermore, various universal features in nonlinear response such as the universality of the efficiency at maximum power were proved in this regime 


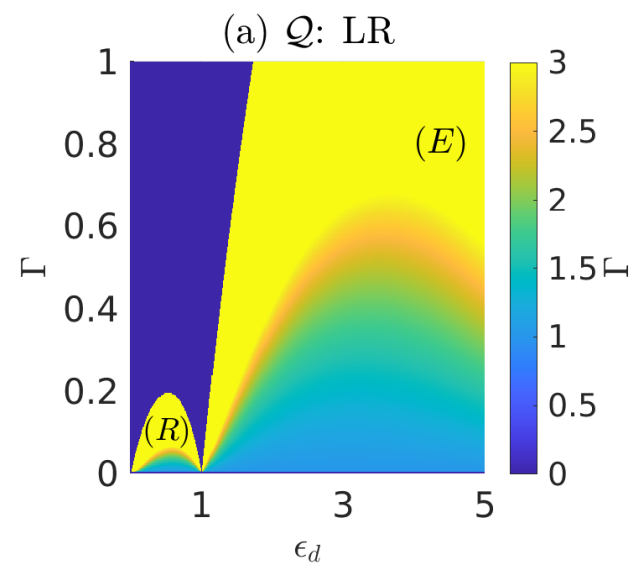

(b) $\mathcal{Q}: \mathrm{FFE}$

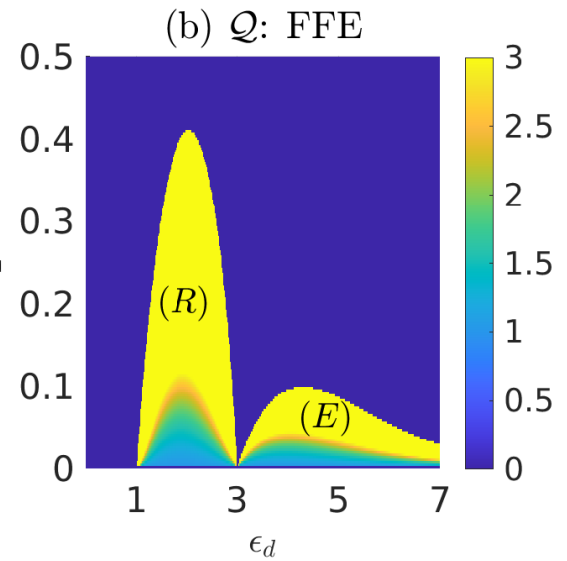

FIG. S3. (Color online) Plot of the ratio $\mathcal{Q}$ for a single dot thermoelectric system within and beyond linear response. We display both the engine $(\mathrm{E})$ and refrigerator $(\mathrm{R})$ regimes for the relevant ranges of $\epsilon_{d}$ and $\Gamma$. (a) Linear response simulations with $\beta_{L}=1.01, \beta_{R}=1, \mu_{L}=0.01, \mu_{R}=0$. (b) Beyond linear response simulations with $\beta_{L}=2, \beta_{R}=1, \mu_{L}=1, \mu_{R}=-1$. We confirm that in both the engine and refrigerator regimes, $\mathcal{Q} \geq 1$. For clarity of presentation, values of $\mathcal{Q}>3$ were assigned the value 3 .

$[2]$.

We now study the ratio between work and heat fluctuations in the TC limit for a thermoelectric engine, arbitrarily far from equilibrium. First, we calculate the average thermodynamic efficiency in the TC limit,

$$
\begin{aligned}
\langle\eta\rangle & =\frac{-\langle\dot{w}\rangle}{\left\langle\dot{q}_{h}\right\rangle} \\
& =\frac{\Delta \mu\left\langle I_{e}\right\rangle}{\left\langle I_{u}\right\rangle-\mu_{R}\left\langle I_{e}\right\rangle}=\frac{\Delta \mu}{\left(\epsilon_{d}-\mu_{R}\right)} .
\end{aligned}
$$

The upper bound for the mean efficiency is given by the Carnot value $\eta_{C}=1-\frac{T_{L}}{T_{R}}$. This can be proved by enforcing the positivity requirement for the average entropy production rate. In steady state, it is defined as $\langle\sigma\rangle=\sum_{i=e, u} A_{i}\left\langle I_{i}\right\rangle$ where $A_{e}=\left(\beta_{R} \mu_{R}-\beta_{L} \mu_{L}\right)$ and $A_{u}=\left(\beta_{L}-\beta_{R}\right)$ are the two thermodynamic affinities corresponding to particle and energy current, respectively [3]. In the TC limit, this reduces to

$$
\langle\sigma\rangle=\left[\beta_{L}\left(\epsilon_{d}-\mu_{L}\right)-\beta_{R}\left(\epsilon_{d}-\mu_{R}\right)\right]\left\langle I_{e}\right\rangle \geq 0,
$$

which is always non-negative. The zero net entropy production limit corresponds to a quasi-static situation, which, interestingly, in TC limit can be achieved without requiring the individual affinities to go to zero. The above condition in the context of thermoelectric engine yields,

$$
\beta_{L}\left(\epsilon_{d}-\mu_{L}\right) \geq \beta_{R}\left(\epsilon_{d}-\mu_{R}\right),
$$

which implies that

$$
\langle\eta\rangle=\frac{\Delta \mu}{\left(\epsilon_{d}-\mu_{R}\right)} \leq 1-\frac{\beta_{R}}{\beta_{L}}=\eta_{C} .
$$

As expected, the mean efficiency of the engine is limited by the Carnot value $\langle\eta\rangle \leq \eta_{C}$.

We now generalize this result for higher order $(n \geq 1)$ cumulants. Since the stochastic energy current goes hand in hand with the stochastic electron flux i.e., $I_{u}=\epsilon_{d} I_{e}$, higher order cumulants satisfy $\left\langle I_{u}^{n}\right\rangle_{c}=\epsilon_{d}^{n}\left\langle I_{e}^{n}\right\rangle$ and $\left\langle I_{q}^{n}\right\rangle_{c}=$ $\left(\epsilon_{d}-\mu_{R}\right)^{n}\left\langle I_{e}^{n}\right\rangle_{c}$ resulting in

$$
\begin{aligned}
\eta^{(n)} & \equiv \frac{\left\langle(-\dot{w})^{n}\right\rangle_{c}}{\left\langle\dot{q}_{h}^{n}\right\rangle_{c}} \\
& =\frac{(\Delta \mu)^{n}}{\left(\epsilon_{d}-\mu_{R}\right)^{n}}=\langle\eta\rangle^{n} \leq \eta_{C}^{n} .
\end{aligned}
$$

Since $\langle\eta\rangle \leq \eta_{C}$ we conclude that in the TC limit, $\eta^{(n)} \leq \eta_{C}^{n}$. This result holds arbitrarily far from equilibrium. 

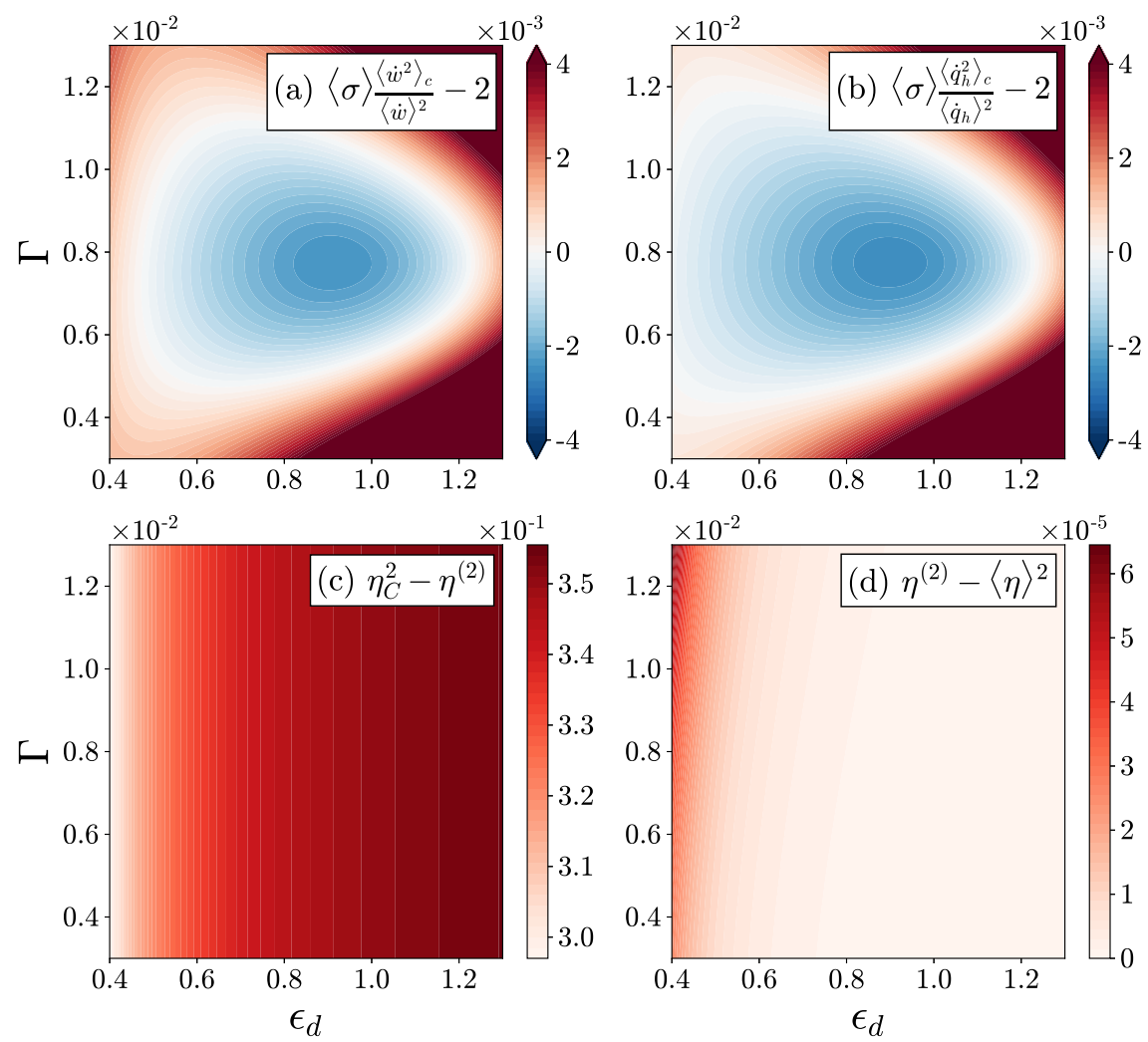

FIG. S4. (Color online) The TUR is violated for the work (a) and heat (b) currents in a serial double-dot thermoelectric engine. However, the upper (c) and lower (d) bounds on $\eta_{\text {eng }}^{(2)}$ are still satisfied. We use degenerate levels with intersite tunneling energy $\Omega=0.005$ and equal hybridization energies to the leads, $\Gamma$. $\beta_{L}=1.0, \beta_{R}=0.4, \mu_{L}=0.1, \mu_{R}=0$.

\section{TUR violation - and the validity of our bounds}

The standard TUR, $\langle\sigma\rangle \frac{\left\langle I^{2}\right\rangle_{c}}{\langle I\rangle^{2}} \leq 2$, can be violated in quantum transport junctions. Specifically for thermoelectric junctions, the TUR can be violated for both work current, $\dot{w}$, and heat current, $\dot{q}_{h}$, in a symmetric serial double-dot thermoelectric system behaving as an engine for a limited range of values of $\epsilon_{d}$ and $\Gamma$ [33]. This behavior is shown in Fig. S4(a)-(b). Here, $\epsilon_{d}$ is the energy of the levels and $\Gamma$ is the (identical) coupling of the left and right dots to the respective metals. The intersite tunneling is denoted by $\Omega$. However, we find that the ratio $\mathcal{Q}$ of relative fluctuations between these two quantities is still greater than 1, thus satisfying the bounds on $\eta^{(2)}$ independently of the TUR itself, see Fig. S4(c)-(d). We reiterate that the proof in the main text for $\mathcal{Q} \geq 1$, translating to $\langle\sigma\rangle \frac{\left\langle\dot{w}^{2}\right\rangle_{c}}{\langle\dot{w}\rangle^{2}} \geq\langle\sigma\rangle \frac{\left\langle\dot{q}_{h}^{2}\right\rangle_{c}}{\left\langle\dot{q}_{h}\right\rangle^{2}}$ for an engine was achieved under linear response. We point out that Fig. S4 displays the validity of this inequality outside this strict regime.

\section{Optimization process for verifying the bounds}

Following the main text we expand here on the stochastic optimization process we are using to verify the bounds beyond linear response. The following procedure is implemented in JAX [4] and uses the ADAM algorithm [43].

We set the biases $\Delta \mu$ and $\Delta T$. We then start at an initial point in parameter space, $\left(\epsilon_{d}^{(i)}, \Gamma^{(i)}\right)$ and aim to minimize the following cost function

$$
f\left(\epsilon_{d}, \Gamma\right)=\left(\eta^{(2)}-\langle\eta\rangle^{2}\right)^{2}+P\left(\left\langle I_{e}\right\rangle\right),
$$

where the first term can be replaced with the equivalent difference for the upper bound, and $P\left(\epsilon_{d}, \Gamma\right)$ is a 
penalty function, which depends on the charge current's direction, and ensures the engine regime. $P\left(\epsilon_{d}, \Gamma\right)=$ $10+\frac{10^{-5}}{10^{3} \operatorname{ReLU}\left(\left\langle I_{e}\right\rangle\right)-10^{-6}}$, where ReLU is a rectifier linear unit. This specific choice of penalty function attempts to ensure a high penalty when the current is positive (outside the engine regime). It is zero for negative currents. Near the (engine-no engine) boundary, the currents assume very small values, which could be $\mathcal{O}\left(10^{-9}\right)$. This issue lead us to set the prefactors as indicated.

Through the optimization process for a specific initial choice $\left(\epsilon_{d}^{(i)}, \Gamma^{(i)}\right)$, a path of points (trajectory) is created. This path can, at times, run away from the desired engine regime. For that, we store the minimal difference, $\eta^{(2)}-\langle\eta\rangle^{2}$ along each path. Since the difference is a smooth function, if it flips sign, we expect that at least one path should have a negative difference in the vicinity of the minima we find within the engine region. Yet, we did not encounter negative differences in the engine domain along any of the paths, hence, we are certain the bounds are obeyed for this choice of biases.

\section{SINGLE AFFINITY LIMIT}

Here we discuss the behavior of the $\mathcal{Q}$ ratio in the single affinity limit. Let us first turn off the affinity corresponding to the input channel, i.e., $A_{1}=0$. One then receives in the linear-response limit,

$$
\mathcal{Q}_{A_{1}=0} \equiv \frac{\left\langle I_{1}\right\rangle^{2}}{\left\langle I_{1}^{2}\right\rangle_{c}} \frac{\left\langle I_{2}^{2}\right\rangle_{c}}{\left\langle I_{2}\right\rangle^{2}}=\frac{L_{12}^{2}}{L_{22}^{2}} \frac{L_{22}}{L_{11}}=\frac{L_{12}^{2}}{L_{22} L_{11}} \leq 1,
$$

where the inequality follows from the positivity of entropy production in a spontaneous process. This result implies that the relative fluctuation or the precision of the current related to the applied bias $\left(I_{2}\right)$ is always upper bounded by the corresponding precision for the current of the coupled phenomena $\left(I_{1}\right)$. This further indicates that in the linear response limit, the TUR ratio for a current under its bias, is always upper bounded by the corresponding TUR ratio for the coupled current i.e., $\langle\sigma\rangle \frac{\left\langle I_{2}^{2}\right\rangle_{c}}{\left\langle I_{2}\right\rangle^{2}} \leq\langle\sigma\rangle \frac{\left\langle I_{1}^{2}\right\rangle_{c}}{\left\langle I_{1}\right\rangle^{2}}$. Similarly, in the opposite limit, i.e., for $A_{2}=0$, following similar steps, it is easy to see that $Q_{A_{2}=0} \geq 1$ i.e., we reach the same conclusion: the current that corresponds to the applied bias is upper bounded by the relative fluctuation of the coupled current.

\section{QUANTUM ABSORPTION REFRIGERATORS}

We describe here the procedure for calculating heat currents and their fluctuations for multi-level QARs in the incoherent transport limit. Additional details can be found in [48]. More advanced simulations, taking into account quantum coherences were performed in Ref. [49].

In our setup, a multi-level system is coupled to three heat baths $(b=w, h, c)$. The objective of interest is the characteristic function, $|Z(\chi, t)\rangle$. For a three-bath problem it is sufficient to consider two counting parameters $\chi=$ $\left(\chi_{c}, \chi_{w}\right)$ when studying properties in steady state, given conservation of energy. The characteristic function satisfies a first-order differential equation,

$$
\frac{d|Z(\chi, t)\rangle}{d t}=\hat{W}(\chi)|Z(\chi, t)\rangle
$$

For the three-level model presented in the main text, Fig. 3a, the rate matrix is

$$
\hat{W}(\chi)=\left(\begin{array}{ccc}
-k_{1 \rightarrow 2}^{c}-k_{1 \rightarrow 3}^{h} & k_{2 \rightarrow 1}^{c} e^{-i \chi_{c} \theta_{c}} & k_{3 \rightarrow 1}^{h} \\
k_{1 \rightarrow 2}^{c} e^{i \chi_{c} \theta_{c}} & -k_{2 \rightarrow 1}^{c}-k_{2 \rightarrow 3}^{w} & k_{3 \rightarrow 2}^{w} e^{-i \chi_{w} \theta_{w}} \\
k_{1 \rightarrow 3}^{h} & k_{2 \rightarrow 3}^{w} e^{i \chi_{w} \theta_{w}} & -k_{3 \rightarrow 2}^{w}-k_{3 \rightarrow 1}^{h}
\end{array}\right)
$$

Here, we count the three levels (bottom to top) by $1,2,3$, with energies $\epsilon_{1,2,3}=\left(0, \theta_{c}, \theta_{w}\right)$. Assuming that the baths comprise collections of harmonic oscillators, which are coupled to the quantum system through the displacements of the oscillators, the rate constant due to the $b$ bath is given by the product

$$
k_{i \rightarrow j}^{b}=J_{b}\left(\epsilon_{j}-\epsilon_{i}\right) n_{b}\left(\epsilon_{j}-\epsilon_{i}\right) .
$$

Here, $n_{b}(\Delta)=\left(e^{\beta_{b} \Delta}-1\right)^{-1}$ is the Bose-Einstein distribution function and $J_{b}(\Delta)=\gamma_{b} \Delta e^{-|\Delta| / \Lambda}$ is the spectral density function of the $b$ bath. For simplicity, we assume Ohmic spectral functions and take the cutoff frequency to be large, $\Lambda \gg \theta_{c, w}, 1 / \beta$. 
For the four-level model as presented in Fig. 3b of the main text, the rate matrix is

$$
\hat{W}(\chi)=\left(\begin{array}{cccc}
-k_{1 \rightarrow 2}^{c}-k_{1 \rightarrow 3}^{c}-k_{1 \rightarrow 4}^{h} & k_{2 \rightarrow 1}^{c} e^{-i \chi_{c}\left(\theta_{c}-g\right)} & k_{3 \rightarrow 1}^{c} e^{-i \chi_{c}\left(\theta_{c}+g\right)} & k_{4 \rightarrow 1}^{h} \\
k_{1 \rightarrow 2}^{c} e^{i \chi_{c}\left(\theta_{c}-g\right)} & -k_{2 \rightarrow 1}^{c}-k_{2 \rightarrow 4}^{w} & 0 & k_{4 \rightarrow 2}^{w} e^{-i \chi_{w}\left(\theta_{w}+g\right)} \\
k_{1 \rightarrow 3}^{c} e^{i \chi_{c}\left(\theta_{c}+g\right)} & 0 & -k_{3 \rightarrow 1}^{c}-k_{3 \rightarrow 4}^{w} & k_{4 \rightarrow 3}^{w} e^{-i \chi_{w}\left(\theta_{w}-g\right)} \\
k_{1 \rightarrow 4}^{h} & k_{2 \rightarrow 4}^{w} e^{i \chi_{w}\left(\theta_{w}+g\right)} & k_{3 \rightarrow 4}^{w} e^{i \chi_{w}\left(\theta_{w}-g\right)} & -k_{4 \rightarrow 1}^{h}-k_{4 \rightarrow 2}^{w}-k_{4 \rightarrow 3}^{w}
\end{array}\right)
$$

We count the levels 1 to 4 from bottom to top, with energies $\epsilon_{1,2,3,4}=\left(0, \theta_{c}-g, \theta_{c}+g, \theta_{c}+\theta_{w}\right)$.

The cumulant generating unction (CGF) is given in terms of the characteristic function,

$$
G(\chi)=\lim _{t \rightarrow \infty} \frac{1}{t} \ln \langle I \mid Z(\chi, t)\rangle,
$$

with $\langle I|$ a unit vector $(\langle I=\langle 1111|$ for the 4-level QAR). To obtain the CGF, we diagonalize $\hat{W}(\chi)$ and select the eigenvalue that dictates the long-time dynamics - this is the eigenvalue with the smallest magnitude for its real part. Once we reach the CGF, the currents and the second cumulants are given by $(b=c, w)$

$$
\begin{aligned}
\left\langle I_{b}\right\rangle & =\left.\frac{\partial G}{\partial\left(i \chi_{b}\right)}\right|_{\chi=0}, \\
\left\langle I_{b}^{2}\right\rangle_{c} & =\left.\frac{\partial^{2} G}{\partial\left(i \chi_{b}\right)^{2}}\right|_{\chi=0} .
\end{aligned}
$$

This procedure can be done analytically for the threelevel QAR. In the main text we obtain the current and its fluctuations numerically: we diagonalize numerically the rate matrix $\hat{W}(\chi)$ for a range of $\chi$. The cumulants are then obtained using finite differences.

[1] T. E. Humphrey, R. Newbury, R. P. Taylor, and H. Linke, Reversible Quantum Brownian Heat Engines for Electrons, Phys. Rev. Lett. 89, 116801 (2002).

[2] M. Esposito, K. Lindenberg, and C. Van den Broeck, Thermoelectric efficiency at maximum power in a quantum dot, Europhysics Letters 85, 60010 (2009).

[3] The chemical potential difference $\Delta \mu$ is attached here to the affinity, with the charge current as the corresponding current. If the output current is the power, $-\langle\dot{w}\rangle=\Delta \mu\left\langle I_{e}\right\rangle$, then the corresponding affinity should be stripped of $\Delta \mu$.

[4] J. Bradbury et al., JAX: composable transformations of Python+NumPy programs, http://github.com/google/jax 